Ziemke, D.; Metzler, S.; Nagel, K.

\title{
Bicycle traffic and its interaction with motorized traffic in an agent-based transport simulation framework
}

Journal article | Accepted manuscript (Postprint)

This version is available at https://doi.org/10.14279/depositonce-8348

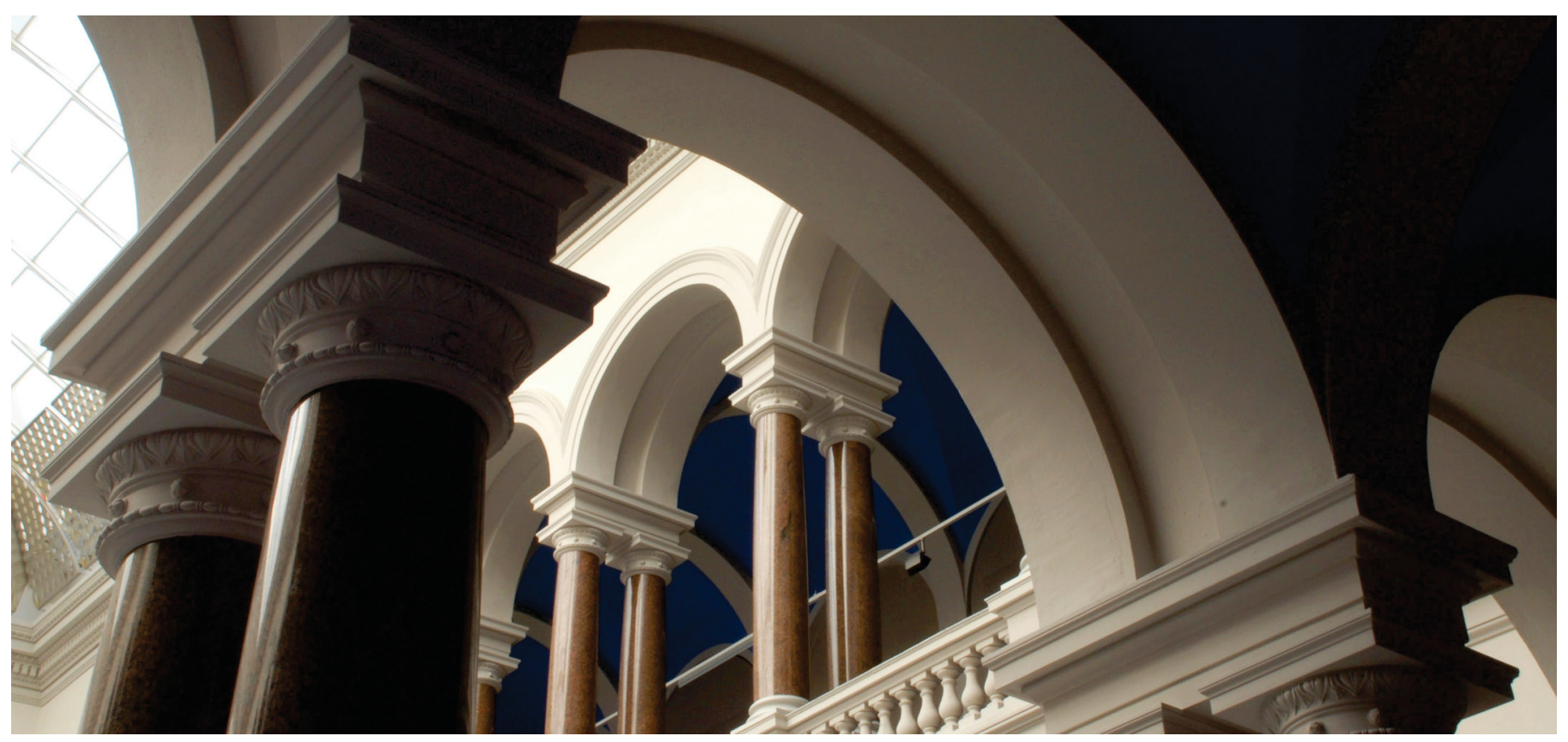

Ziemke, D.; Metzler, S.; Nagel, K. (2018). Bicycle traffic and its interaction with motorized traffic in an agent-based transport simulation framework. Future Generation Computer Systems, 97, 30-40. https:// doi.org/10.1016/j.future.2018.11.005 


\title{
Bicycle traffic and its interaction with motorized traffic in an agent-based transport simulation framework
}

\author{
Dominik Ziemke ${ }^{a, *}$, Simon Metzler ${ }^{\mathrm{a}}$, Kai Nagel $^{\mathrm{a}}$ \\ ${ }^{a}$ Technische Universität Berlin, Transport Systems Planning and Transport Telematics, \\ Salzufer 17-19, 10587 Berlin, Germany
}

\begin{abstract}
Cycling as an inexpensive, healthy, and efficient mode of transport for everyday traveling is becoming increasingly popular. While many cities are promoting cycling, it is rarely included in transport models and systematic policy evaluation procedures. The purpose of this study is to extend the agent-based transport simulation framework MATSim to be able to model bicycle traffic more realistically. The network generation procedure is enriched to include attributes that are relevant for cyclists (e.g. road surfaces, slopes). Travel speed computations, plan scoring, and routing are enhanced to take into account these infrastructure attributes. The scoring, i.e. the evaluation of simulated daily travel plans, is furthermore enhanced to account for traffic events that emerge in the simulation (e.g. passings by cars), which have an additional impact on cyclists' decisions. Inspired by an evolutionary computing perspective, a randomizing router was implemented to enable cyclists to find realistic routes. It is discussed in detail why this approach is both feasible in practical terms and also conceptually consistent with MATSim's co-evolutionary simulation approach. It is shown that meaningful simulation results are obtained for an illustrative scenario, which indicates that the developed methods will make real-world scenarios more realistic in terms of the representation of bicycle traffic. Based on the exclusive reliance on open data, the approach is spatially transferable.
\end{abstract}

\footnotetext{
${ }^{*}$ Corresponding author

Email address: ziemke@vsp.tu-berlin.de (Dominik Ziemke)
}

Preprint submitted to Elsevier

June 26, 2018 
Keywords: cycling, bicycle traffic, transport simulation, agent-based modeling, agent-based simulation, open data

2010 MSC: 00-01, 99-00

\section{Introduction}

Cycling as an inexpensive, fast, healthy, quiet, energy-efficient, limitedly land-consuming, and enjoyable mode of transport for everyday travel is becoming increasingly popular in many regions of the world [1, 2, 3, 4. Aware of the 5 societal, environmental, economic, and public-health-related problems that motorized traffic has contributed to [5] and recognizing the benefits of cycling, cities around the world are promoting the use of the bicycle for everyday travel [6]. The encouragement of cycling is increasingly included into plans for travel behavior change [1. In Berlin, for instance, the current city-wide modal share of cycling ranges at $13 \%$, with increasing tendency [7]. At the same time, $64 \%$ of all trips in Berlin are shorter than 5km, which illustrates the vast growth potential that cycling still has [7. Currently, a new mobility law ('Mobilitätsgesetz') is scheduled to be passed whose aim is to make cycling, but also walking and using public transport, safer and more comfortabl $\rrbracket^{1}$

Next to other policies, the implementation of a good cycling infrastructure appears to be an important prerequisite for fostering further growth in cycling 3. Many projects ranging from local additions of bicycle lanes and improved intersection designs to ambitious larger-scale projects like the Radschnellweg Ruhr, an about 100km-long bicycle highway that is sought to shift parts of commuter traffic to bicycles in the Ruhr region in Germany ${ }^{2}$ or the Emscher-Weg, a large-scale conversion of former industry sites along the river Emscher into a 100-km-long riverside bicycle rout 3 are currently discussed and

\footnotetext{
1 Cf. https://www.berlin.de/senuvk/verkehr/mobilitaetsgesetz/index_en.shtml, last accessed on 16 June 2018.

2 Cf. http://www.rs1.ruhr last accessed on 30 May 2018.

3 Cf. http://www.emscher-weg.de/route/emscher-weg/, last accessed on 24 June 2018.
} 
implemented. While some cities like Copenhagen or Amsterdam have been forerunners in promoting cycling, in many other places limited information about the preferences of cyclists has been mentioned as obstacles to an effective investments in bicycle infrastructure [6].

Transport models are recognized as an important tool to support the effective planning of transport systems and as a means to evaluate proposed policies in a structured and systematic fashion. While transport models are state-of-thepractice in terms of motorized transport and public transport, this is not the case for the mode of cycling [3].

In this study, the agent-based transport simulation framework MATSim [8] is used. The most simple way to include a mode of transport into a MATSim transport simulation scenario is by 'teleportation'. If a person (agent) choses 35 a 'teleported' mode in the simulation, they are simply relocated from their previous activity location to the next activity location without any network interaction, but with a time delay that reflects real travel times. This approach is usually only applied for modes that are included as alternatives to those modes that are in the actual focus of analysis of a given scenario. Of course, 40 accuracy and, in particular, policy sensitivity of the teleportation approach are limited.

An improvement over the teleportation approach for modeling bicycle and walk traffic was implemented by Dobler and Lämmel [9] who take into account attributes of the person as well as link gradients to compute realistic travel 45 speeds for cyclists and pedestrians. Like for teleportation, their approach does, however, not contain an explicit simulation of the cycling agents on the network. This is justified by the observation that congestion is rare for cyclists compared to motorized traffic. As such, their approach does not allow for a simulation of the interaction of different modes on the network like it is done in the present 50 study.

In the current approach, by contrast, the movement of cyclists on the representation of the physical network is explicitly simulated. To model bicycle traffic realistically, various aspects of mobility that are relevant for cyclists are 
taken into account. This includes both aspects of the infrastructure as well as the interaction of road users as it emerges in the transport system. In a preceding study [10, it has been shown how the routing module of MATSim can be amended to observe cyclist-relevant properties of the infrastructure like the existence of bicycle lanes or bicycle tracks as well as different road surface types. The present paper builds on that work and extends it by also adjusting MATSim's scoring function. This is necessary to be able to run a simulation over multiple iterations, which, in turn, is necessary to be able to conduct policy studies and to simulate the interaction of different road users on the network. In fact, cycling agents might choose to avoid routes where they have experienced a lot of motorized traffic, since the presence of cars tends to make cycling more uncomfortable and potentially less safe, in particular if there is no dedicated cycling infrastructure [5, 11]. This study explains the incorporation of such events into MATSim's scoring function in addition to the consideration of additional infrastructure attributes. By this, full advantage is taken of the agent-based nature of the MATSim transport simulation, which allows to follow individual synthetic travelers during the whole simulated day. It is discussed how the approach can be extended to account for personal and vehicle-specific attributes that influence the behavior of cyclists [12].

The remainder of this paper is structured as follows: Section 2 summarizes a literature review, which identifies attributes of infrastructures and aspects of mobility that are relevant for cyclists, especially with respect to route choice and travel speed. Section 3 introduces the agent-based transport simulation framework MATSim and the corresponding input data preparation including the retrieval of cyclist-relevant infrastructure attributes based on open data. This sections also describes the adaptations in terms of travel time computation, utility computation, routing, and scoring that were made in order to be able to simulate bicycle traffic more realistically. Section 4 presents results for an illustrative scenario, in which the implemented adaptations are analyzed. In section 5 the methodology, the findings, and the potential of the approach are discussed and an outlook for possible further steps is given. 


\section{Literature review}

Choices of cyclists, in particular cyclists' route choice, have been surveyed in various studies, mostly in terms of stated-preference analyses [13, 3, 5, 2, but recently also in terms of GPS-based revealed-preference analyses [1, 6, 11. Most studies report that their results are partially in agreement with findings from previous studies, while other findings stand in contradiction [6, 3, 1 .

Travel time and route length have generally been found to be important factors in route selection [5, 6, 11]. Studies also largely agree that cyclists tend to avoid slopes [1, 6, 2, 11, 12. Different authors state different specific definitions of slope to be most significant, ranging from average slope and maximum slope to summations of gains and losses in height on a route. Broach et al. 11. explicitly research different definitions of slope and find route lengths within defined categories of average positive slope to be most significant.

There is also agreement that cyclists strongly prefer a continuous cycling infrastructure [5, 2]. Hood et al. 6] specifically find that cyclists prefer bicycle lanes over other types of bicycle infrastructure. Menghini et al. 1] emphasize the importance of direct and marked routes for cyclists, which is confirmed by other studies [14. Bai et al. 12] add that the existence of bicycle lanes significantly increases the comfort perception of cyclists, which can also be achieved by a physical separation between motorized traffic and bicycle lanes.

Cyclists also generally try to avoid signal-controlled junctions [1, 5, 11, 3. Additionally, some stated-preference studies find the type of parking along cycling facilities [5, 2, and existence of bus stops [2] to be influential.

Next to travel time, Sener et al. 5] find motorized traffic volumes to be one of the most important attributes in bicycle route choice. Broach et al. 11] confirm that cyclists are highly sensitive to traffic volumes and add that the existence of bicycle lanes helps to offset the negative effects of adjacent traffic. They find that streets with a high traffic volume and a dedicated bicycle lane are perceived about as attractive (or unattractive) as streets with low traffic volumes and no dedicated bicycle lane. While Li et al. 2] confirm the 
Milakis and Athanasopoulos [3] find it to be less relevant.

Studies also agree that pavement surface conditions and riding smoothness are important factors [14, 15, 3. To be able to include such aspects into a model, it is important to know how cyclists evaluate different road surface a bicycle to quantify rolling resistance - find that asphalt is associated with the lowest rolling resistance and level of vibrations, followed by concrete slabs and self-binding gravel. The highest rolling resistance is measured for cobblestones. Its quantitative value differs significantly from that of the three other attached to a bicycle - find old cobblestone pavements to be associated with significantly higher vibrations and, thus, rolling resistance to be higher than on other types of pavement including asphalt (new, worn, and uneven), concrete (uneven and interlocking) and unpaved roads. Ayachi et al. 13, based on more comfortable than other types of roads.

Finally, various attributes of the cyclists (e.g. age, gender) and the type of the bicycle are relevant [12]. Related to this, Hebenstreit and Fellendorf [17] use user-group-specific bicycle friendliness values for different links. The purpose of find that steep hills are disfavored more strongly by women and commuters, i.e. riders whose trip purpose is going to (or returning from) work.

In a recent study based on GPS observations, Flügel et al. 18 develop a model that describes cyclists' travel speeds based on attributes related to the cyclist, the trip, and the infrastructure. They find that cyclists' speeds decrease roughly linearly with increasing slopes: At a gradient of $9 \%$, the average cyclist rides $42.7 \%$ slower than on level terrain.

In summary, modeling bicycle traffic and cyclist's route choice is a challenging task. Route choices of users of motorized vehicles are mostly modeled based 
to minimize travel time or generalized costs whose components (e.g. monetary cost or distance) are typically strongly correlated with travel time (an exception is toll; cf. Nagel et al. [19]). As discussed before, cyclists, by contrast, typically also take into account objectives that are not necessarily correlated with travel time (e.g. riding comfort, gradients, and presence of motorized traffic). A bit philosophically, one could say that a car 'homogenizes' the travel behavior of transport users who themselves have much more heterogeneous characteristics. In cycling, by contrast, a large portion of this heterogeneity of transport users directly comes into effect. Therefore, to model bicycle traffic realistically, these relevant attributes need to be taken into account in a reasonable way.

\section{Methodology}

To simulate bicycle traffic in this study, MATSim [8], an agent-based demand adaptation and traffic assignment model, is used. In MATSim, each person is resolved individually as an agent and has one or more plans. A plan is a chain of activities (e.g. home-work-shop-home), including locations and activity end times. Activities at different locations are connected by trips, adhering to the notion of transport as a derived demand. MATSim is based on a co-evolutionary algorithm, where each iteration consists of the following three steps: Traffic simulation, scoring, and replanning (cf. figure 1).

In the traffic simulation step (also referred to as mobility simulation (mobsim) or network loading), the demand for transport is simulated on the physical network. The selected plans of all agents are executed simultaneously. The default physical simulation is a queue model, in which every roadway segment (link) is modeled as a first-in-first-out (FIFO) queue. This computationally efficient design makes MATSim suitable for large-scale scenario (e.g. scenarios which include whole metropolitan regions).

After the traffic simulation, the choice processes (decision making) that travelers undertake, partially in reaction to what they experience while traveling in the synthetic reality, are simulated. This consists of scoring and replanning and 


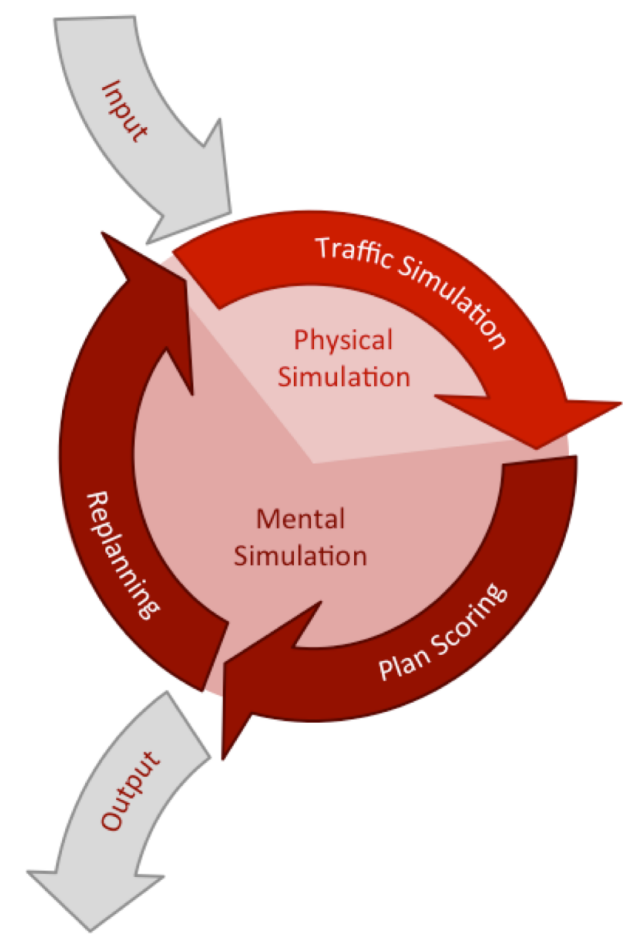

Figure 1: MATSim simulations steps.

is also referred to as mental simulation. In the scoring step, agents compute, based on the events of the full simulated day and the notion of utility, a score $4^{4}$

$$
S_{\text {plan }}=\sum_{q=0}^{N-1} S_{a c t, q}+\sum_{q=0}^{N-1} S_{\text {trav }, q},
$$

where $S_{a c t(q)}$ is the utility that the agent receives from performing activity $q, S_{\text {trav, } \operatorname{mode}(q)}$ is the (typically negative) utility that the agent receives from making the trip (leg) after activity $q$, and $N$ is the number of activities.

The utility $S_{a c t, q}$ of performing activity $q$ is computed as

$$
S_{a c t, q}=\beta_{d u r} \cdot t_{t y p, q} \cdot \ln \left(\frac{t_{d u r, q}}{t_{0, q}}\right)
$$

\footnotetext{
${ }^{4}$ It might be worth mentioning that score is the technical term in the MATSim context for what is in economics commonly referred to as utility. Also cf. Nagel et al. 20].
} 

duration, $t_{t y p, q}$ is the typical duration of an activity of the type of activity $q$, $t_{d u r, q}$ is the duration of activity $q$, and $t_{0, q}$ is the duration from which on a positive utility can be obtained ${ }^{5}$ As described by Nagel et al. [20, the utility of performing an activity can include additional other components, e.g. for having to wait or for arriving too late at a time-constrained activity.

The (typically negative) utility $S_{\text {trav }, q}$ of traveling leg $q$ in equation 1 is computed as

$$
S_{\text {trav }, q}=C_{\operatorname{mode}(q)}+\beta_{\text {trav }, \operatorname{mode}(q)} \cdot t_{t r a v, q}+\beta_{d, \operatorname{mode}(q)} \cdot d_{t r a v, q}
$$

where $C_{\operatorname{mode}(q)}$ is a mode-specific constant, $\beta_{\text {trav }, \operatorname{mode}(q)}$ is the marginal utility of time spent traveling by $\operatorname{mode}(q)$ that is used for leg $q, t_{\text {trav }, q}$ is the travel time on leg $q, \beta_{d, \operatorname{mode}(q)}$ is the marginal utility of distance when traveling by mode $(q)$, and $d_{\text {trav }, q}$ is the distance traveled on leg $q$. The distance component is in MATSim's default utility function related to the utility of monetary expenditures [20, equation 3.4]. Since it is assumed that for cycling marginal monetary costs of traveling are negligible, the monetary utility component is not included in equation 4 for better comprehensibility and brevity.

Taking into account this score, agents decide in the replanning step of the iteration which plan to execute in the traffic simulation of the next iteration. Alternatively, agents may generate a new plan by modifying a copy of one randomly selected existing plan of their plan choice set. Modifications may be done with respect to various choice dimensions (e.g. routing or time choice) by

5 The computation of $t_{0, q}$ can be specified as either uniform or relative. In this study, the setting typicalDurationScoreComputation = relative is chosen. As such, $t_{0, q}$ is defined by the expression $t_{0, q}:=t_{t y p, q} \cdot \exp \left(-1 /\right.$ prio $\left._{q}\right)$, where prio $_{q}$ is a configurable parameter. This allows to re-write equation 2 as

$$
S_{a c t, q}=\beta_{d u r} \cdot t_{t y p, q} \cdot \ln \left(t_{d u r, q} / t_{t y p, q}\right)+\frac{\beta_{d u r} \cdot t_{t y p, q}}{p r i o_{q}} .
$$

At the typical duration (i.e. $t_{d u r, q}=t_{t y p, q}$ ), the first term is zero, and thus all activity types with the same value of prio $_{q}$ generate the same relative score of $\beta_{d u r} /$ prio $_{q}$ per hour at their typical durations. 
applying (innovative) strategy modules. When computing a new route, agents use Dijkstra's algorithm [21. The generalized cost $C_{t r a v, q}$ of a route (or leg) $q$ is, in MATSim's default version, computed as

$$
C_{\text {trav }, q}=-\left(\beta_{\text {trav }, \text { mode }(q)}-\beta_{\text {dur }}\right) \cdot t_{\text {trav }, q}-\beta_{d, \operatorname{mode}(q)} \cdot d_{\text {trav }, q} .
$$

$-\beta_{\text {trav }, \operatorname{mode}(q)} \cdot t_{\text {trav }, q}$ is the direct disutility of travelling; since $\beta_{\text {trav }, \operatorname{mode}(q)}$ is typically negative, this term is typically a positive cost contribution. $-\left(-\beta_{d u r}\right)$. $t_{\text {trav }, q}$ approximates the effect of having to make activities shorter and thus losing utility when travel takes longer. $-\beta_{d, \operatorname{mode}(q)} \cdot d_{\text {trav }, q}$ is the disutility of distance; since $\beta_{d, \operatorname{mode}(q)}$ is typically negative, this term is again a positive cost contribution.

In terms of the components that are included in equation 5 , routing is consistent with scoring. As can be seen by closer inspection, however, the numeric values of utility computed for scoring (cf. equation 1) and routing (cf. equation 5) will in general not be the same. This is due to the logarithmic component of the scoring equation (cf. equation 2). Accordingly, the ratio between the utility components, in particular that of the cost of time, varies between different users and different trips. Conceptually, this means that agents have different values of travel time savings (VTTS) 22. The router, by contrast, assumes a constant ratio between the marginal utilities.

Intuitively, one could, therefore, argue that it should be tried to increase the consistency between the routing module (and potentially other innovative modules) and scoring. This is, however, associated with various issues: To achieve consistency, the actual activity times $t_{d u r, q}$ would need to be known when applying the router. However, this is - unless activity times are fixed by simulation configuration - not possible as these times are emergent from the traffic simulation and, therefore, only known once the traffic simulation of an iteration is finished.

Furthermore, even if a correct linearization of the scoring function was achieved and the factors in equation 5 correctly adapted, the router (in its default implementation) operates on travel times that are stored per link in 

scoring function may not find the best solution. Nagel et al. [22] discuss further conceptual issues related to achieving consistency between routing and scoring.

Most importantly, however, the achievement of such a consistency is not in line with the fundamental concept of MATSim's co-evolutionary algorithm.

220 In fact, it is intended that the innovative strategy modules (e.g. the routing module) are conceptually independent from scoring. Scoring is meant to be the 'intelligent' component of the simulation of the decision making process of agents and has all relevant information of the synthetic reality, while innovation can be based on arbitrary (mental) models of that system [22]. The relevant goal is that routing and scoring act on the simulation in such a way that, over the course of multiple iterations, a realistic representation of real-world traffic emerges in the simulation. To achieve this, the routing module can, in fact, be quite 'unsophisticated' and, for instance, be largely based on randomness, as long as it manages to find sufficiently diverse travel options.

Considering an evolutionary computing perspective to address this problem, Nagel et al. 22] suggest to increase the variability of the solutions that the router generates by varying the ratio between factors of the components of the utility function used for routing (cf. equation 5). For a tolling study for Gauteng in South Africa, it was, indeed, found that such a randomizing router generates 'better' solutions than a manually calibrated, deterministic router [22].

After the router has generated solutions, which are then simulated in the synthetic reality, the scoring function will - based on detailed knowledge of all the events that occurred in the simulation - compute a score. This score will eventually determine which alternatives remain in the choice set and which ones are soon to be dropped.

In particular with respect to modeling the interactions of cyclists with motorized traffic, it is clear that the relevant events (e.g. number of passing cars on a given link) only emerge during the simulation of the transport system. Making these information available to the router is computationally not straightforward and also appears counterintuitive. 
In summary, a randomizing routing approach appears as a good solution as it significantly reduces both the consistency burden of the software system and the level of detail that the innovative module needs to have about the problem (e.g. the necessary time resolution of the routing module) [22]. In particular,

\subsection{Data}

In general, a MATSim network consists of nodes and links. Nodes store coordinates, while links have a start and an end node, a free-flow speed, a link length, a flow capacity, a storage capacity, and allowed modes. OpenStreetMap [OSM, study, additional information are extracted from OSM, which describe properties of the infrastructure that are relevant for the decisions of cyclists.

OSM objects have tags, which are key-value pairs. Roads are represented by way objects, which can, besides others, possess the tags highway and cycleway. Additional information like bicycle-specific restrictions can be captured from the tag bicycle. As such, a main road with a bicycle lane will be tagged as highway=? and cycleway=lane. In case the cycling infrastructure is located on the sidewalk, the road will have the tag cycleway=track. A standalone bicycle track away from roads for motorized traffic is tagged as highway=cycleway.

The attribute smoothness represents an evaluation of the surface, ranging 
from excellent to impassable. It provides the information that is required to evaluate riding comfort (cf. section 2). However, only $12 \%$ of all links in Berlin possess this attribute. Therefore, it does currently not appear reasonable to base a model on this attribute. As a proxy, the attribute surface, which reflects the type of pavement surface, can be used. In Berlin, for instance, $58 \%$ of all links are provided with a surface tag. Additionally, some highway types are assigned with defaults (e.g. primary highways are assumed to always be asphalt roads), such that the surface type of these roads can be easily inferred. As a result, the surface of most links can be identified by OSM.

\subsubsection{Data on elevation and gradients}

As pointed out in section 2 road gradients (slopes) are an important determinant of a cyclist's route choice. Because of the absence of such information in OSM, a digital elevation model (DEM) is used. Broadly, there are two types of DEMs:

(1) Digital surface models (DSM) are mostly created based on satellite imaging and reflect the surface of the earth including all objects on it, e.g. buildings and trees. Some DSMs like SRTM (Shuttle Radar Topography Mission), provided by NASA, are openly available. As DSMs are, however, not able to capture the surface of the bare ground, these models are not suited for the task at hand as their use would, for instance, create unrealistic slopes in the vicinity of larger buildings.

(2) Digital terrain models (DTM), by contrast, represent the ground surface of the earth without any objects on it. They are created by photogrammetric measurement using aerial picturing and laser scanning, but are rarely openly available. There are, however, algorithms which are able to compute a DTM from a high resolution DSM. In this study, EU-DEM [European Digital Elevation Model, 25] is used, which is a hybrid of SRTM and ASTER (Advanced Spaceborne Thermal Emission and Reflection Radiometer, also provided by NASA) data. By this combination, a high number of artifacts could be removed. EU-DEM is free to download and has a resolution of $25 \mathrm{~m}$. For Berlin, 
a test against SRTM data confirmed that unrealistic slopes due to buildings are significantly decreased.

\subsection{Adaptations to simulate bicycle traffic}

In this section, the adaptations to simulate bicycle traffic in terms of network generation, the computation of travel speeds and travel times, and the utility computations for scoring and routing are described in detail.

\subsubsection{Network generation}

A Bicycle0smNetworkReader was written to create a MATSim network suited to simulate bicycle traffic. It extends the default network generation

315 procedure in two ways:

(1) Besides OSM, the EU-DEM elevation model (cf. section 3.1.1) is used, which provides land elevations in the GeoTiff format. For each node of the tobe-created network, the elevation of its location is queried from the EU-DEM model by an ElevationDataParser that is integrated into the BicycleOsm-

320 NetworkReader. The parsed elevation is added as the third dimension of the node's coordinate in the MATSim network, taking advantage of a recent amendment to MATSim for 3D coordinates [26].

(2) The network is attributed with a number of additional properties parsed from OSM that are relevant for the behavior of cyclists in the transport system 325 (cf. section 2). This takes advantage of another recent enhancement of MATSim, which allows links (and similarly other MATSim objects) to be enriched with user-defined attributes in addition to the usual link properties that were described in section 3.1. For bicycle modeling, the cycleway type and the surface type of a given link are added.

In contrast to default network generation settings, lower-hierarchy ways (e.g. pedestrian zones, paths, service roads) are included into the network as well. Note that despite the enrichment with further link attributes and elevation information, this process is still spatially transferable because only openly available input data (OSM and EU-DEM) are used. 


\subsubsection{Travel speeds and travel times}

To determine the speed by which a bicycle can traverse a given link, it is, first, considered if the link contains some form of dedicated cycling infrastructure. This is the case for standalone cycleways (tag highway=cycleway) or when a road possesses a bicycle lane (tag cycleway=lane) or a bicycle track (tag 340 cycleway=track). For these facilities, it is assumed that - before considering other attributes that may reduce feasible cycling speeds - an infrastructurebased minimum speed of $15 \mathrm{~km} / \mathrm{h}$ is feasible. For instance, the cycling speed in a pedestrian zone, which would have a lower feasible speed otherwise, is increased to $15 \mathrm{~km} / \mathrm{h}$ in case a dedicated bicycle track runs through the pedestrian zone.

Second, the maximum speed of the vehicle is considered. This is essential because the pure infrastructure-based (or regulatory) speed limit would allow higher speeds on most roadways than those that bicyclists can normally reach. For this study, it is assumed that a bicycle can travel $20 \mathrm{~km} / \mathrm{h}$ at most. Based on this, it would also be possible to further distinguish between different types of bicycles (e.g., race bicycles vs. beach cruisers) with their typical maximum speeds [12].

Third, the impact of road gradients is observed. Roughly based on Flügel et al. 18, cf. section 2, speeds are decreased linearly with increasing slope such that the speed on a link with a gradient of $10 \%$ equals $50 \%$ of the original speed at level. Speeds are never reduced to less then $10 \%$ of the corresponding at-grade speed, assuming that a cyclist will get off their bicycle on a steep hill and walk it slowly. Potential downhill speed increases are not taken into account because of their lower impact on cyclists' choices and their comparatively unclear theoretical foundation 6

Finally, speed reductions in relation to riding smoothness, inferred by surface type (cf. section 2), are taken into account. Table 1 describes these speed reductions.

\footnotetext{
${ }^{6}$ A consideration of downhill speed changes is, however, straightforward and can be included once corresponding empirical data become available.
} 
Table 1: Speed factor for different road surface types (not all values shown).

\begin{tabular}{lllll}
\hline Surface & Speed factor & & Surface & Speed factor \\
\cline { 1 - 2 } \cline { 5 - 6 } asphalt & $100 \%$ & & gravel & $70 \%$ \\
concrete_plates & $80 \%$ & & cobblestone & $50 \%$ \\
compressed & $70 \%$ & & sand & $20 \%$ \\
\cline { 5 - 6 } & & &
\end{tabular}

\subsubsection{Scoring function}

In order to account for properties of the route that are particularly relevant for cyclists (as discussed in section 2), but which do not affect travel speeds (cf. section 3.2.2), the scoring of a leg (cf. equation 4) is extended. In addition to the utility components related to travel time and travel distance, new utility components related to the type of infrastructure, riding comfort, and road gradient are added. As such, the utility (score) of traveling leg $q$ by bicycle is computed as

$$
\begin{gathered}
S_{\text {trav }, q}=C_{b}+\beta_{\text {trav }, b} \cdot t_{\text {trav }, q}+\beta_{d, b} \cdot d_{\text {trav }, q} \\
+\sum_{a \in q}\left(\beta_{\text {inf }(a)}+\beta_{\text {comf }(a)}+\beta_{\operatorname{grad}(a)}\right) \cdot \ell_{a}
\end{gathered}
$$

where $C_{b}$ is a bicycle-specific constant, $\beta_{\text {trav }, b}$ is the marginal utility of time spent traveling by bicycle, $t_{t r a v, q}$ is the travel time (by bicycle) on leg $q, \beta_{d, b}$ is the marginal utility of distance by bicycle, $d_{t r a v, q}$ is the distance traveled on leg $q, \beta_{i n f(a)}$ is the marginal utility of distance on the infrastructure type of link $a, \beta_{\operatorname{comf}(a)}$ is the marginal utility of distance on the comfort level of link $a, \beta_{\operatorname{grad}(a)}$ is the marginal utility of distance on the gradient of link $a$, and $\ell_{a}$ is the length of link $a$. Note that the travel time $t_{\text {trav }, q}$ takes into account the speed changes described in section 3.2 .2 To accomplish the scoring defined in equation 6. two alternative approaches were implemented: A leg-based scoring function and a link-based scoring function.

The leg-based scoring function (called BicycleLegScoring) extends MAT375 Sim's default scoring function for travel legs (cf. equation 4, called Charypar- 
NagelLegScoring) and - whenever a travel leg of an agent who goes by bicycle is scored - retrieves the network route. By this, all network links of the route can be identified and the computations in the sum term (i.e. the lower line) of equation 6 be carried out. The concrete functions of these computations are outsourced into a utility class called BicycleUtilityUtils.

The link-based scoring function (called BicycleLinkScoring) uses a different approach. It is based on simulation events that MATSim throws whenever a vehicles enters traffic (at a beginning of a trip), enters a new link (while en route), and leaves traffic (at the end of a trip). By this, all links that an agents traverses are immediately known, including the time (in seconds) of entering and exiting the link. With that information all required computations can be performed. The link-based approach also allows for computations that are based on travel times on specific links. This is in contrast to the leg-based scoring, which does not have the information how much time an agent spent on a specific link. In particular, the link-based scoring approach is required for the consideration of interactions of bicycles with motorized traffic (e.g. cyclists being passed by cars) because it is necessary to know at which exact time which cars and which cyclists where on a specific link. For the concrete computation, the functions of the aforementioned utility class BicycleUtilityUtils are used.

\subsubsection{Scoring of interaction with motorized traffic}

As pointed out in section 2, the amount of motorized traffic on a network section is an important determinant of cyclists' travel behavior, and, in particular, route choice. Cyclists tend to avoid streets with a lot of motorized traffic, which tends to make cycling uncomfortable and potentially unsafe, especially if no dedicated cycling infrastructure is present.

So far, only attributes of the infrastructure, irrespective of the use of these infrastructures by other tripmakers, were considered (cf. section 3.2.3). It is obvious that the number of cars and bicycles on a specific network section at a specific time is emergent from the simulation, i.e. it is only known when that 
As described at the beginning of section 3 it is one of the core features of the agent-based transport simulation framework MATSim to follow individual tripmakers (agents) during their whole day in the simulation. As such, an event that happens in the simulation (e.g. the passing of a cyclists by a car) can be directly taken into account. To achieve this, the bicycle-specific scoring function (cf. equation 6) is extended by the additional term

$$
\sum_{a \in q} \beta_{m o t o r} \cdot n_{m o t o r(a, \Delta t)}
$$

where $\beta_{\text {motor }}$ is the marginal utility of an interaction of a cyclist with a motorized vehicle, and $n_{\text {motor }(a, \Delta t)}$ is the number of interactions with a motorized vehicle on link $a$ during time period $\Delta t$, which is the time that the cyclist needs to traverse link $a$. 7 As explained in section 3.2 .3 , it is necessary to use the link-based version of the new bicycle-specific scoring function (i.e. BicycleLinkScoring) to be able to add this additional scoring component because only the link-based implementation has complete information regarding which cars and which cyclists travel on a specific link at a specific time.

\subsubsection{Routing module}

As discussed at the beginning of section 3, an approach that varies the ratio between factors of the components of the utility computation is proposed to generate a sufficiently large selection of potential travel option that can then be 'tried out' by agents in the simulation. As explained for MATSim's default leg scoring function (cf. equation 4), a randomizing router can be derived by including all those utility components that are also part of the scoring function (cf. section 3.2.3) and by randomizing the marginal utility values of these utility component (except the first one, i.e. the marginal utility of time). This provides a sufficient exploration of the search space of possible travel options (here:

\footnotetext{
${ }^{7}$ Note that this simplistic approach assumes that every car that travels on the link during time period $\Delta t$ passes the cyclist. This inaccuracy will resolve once this approach is integrated with a recent enhancement of MATSim to explicitly simulate vehicle passings [27].
} 
routes), which is particularly critical in the bicycle context, where - as pointed out in section 2- the correlation between relevant properties (e.g. route length, comfort, gradients) to travel time is much weaker than in routing tasks for other modes of transport [4]. Accordingly, the utility function for the bicylce router becomes

$$
\begin{gathered}
C_{\text {trav }, q}=\hat{\beta}_{\text {trav }, b} \cdot t_{\text {trav }, q}+\hat{\beta}_{d, b} \cdot d_{\text {trav }, q} \\
+\sum_{a \in q}\left(\hat{\beta}_{\text {inf }(a)}+\hat{\beta}_{\operatorname{comf}(a)}+\hat{\beta}_{\operatorname{grad}(a)}\right) \cdot \ell_{a},
\end{gathered}
$$

where, for instance, the value of the randomized marginal utility of distance $\hat{\beta}_{d, b}$ is drawn from a log-normal distribution and realized as

$$
\hat{\beta}_{d, b}=\beta_{d, b} \cdot \frac{\exp (\sigma \cdot Z)}{\exp \left(\sigma^{2} / 2\right)},
$$

where $Z$ is drawn from a Gaussian distribution with mean zero and variance one using Java's nextGaussian() method. $\sigma$ is a width parameter. The log-normal distribution was chosen because it contains values from 0 to $\infty$. The denominator $\exp \left(\sigma^{2} / 2\right)$ effects that the expectation value of equation 9 is $\beta_{d, b}[22$. The three marginal utilities of infrastructure $\beta_{\text {inf }(a)}$, comfort $\beta_{\text {comf }(a)}$, and gradient ${ }_{420} \beta_{\operatorname{grad}(a)}$ are randomized analogously, with new draws of the random variable $Z$. As such, the randomized marginal utilities are agent- and iteration-specific, i.e. drawn once per agent in each iteration.

The randomized marginal utility of traveling in equation 6 is computed as

$$
\hat{\beta}_{\text {trav }, b}=\beta_{\text {trav }, b} \cdot(1+X)
$$

where $X$ is drawn from a Gaussian distribution with mean zero and standard deviation 0.05 . This is an additional randomization compared to the randomizing router introduced by Nagel et al. [22]. The variability this creates is, however, much lower than that of the randomization of the other scoring components.

This additional randomization is required because those routes that are good options because of low volumes of motorized traffic might never be found by the router otherwise as there is no component in the utility function of the router 
that takes these properties into account. Based on the described additional randomization, the search space, which otherwise operates between time-, distance, infrastructure-, comfort-, and gradient-optimal routes is further extended so that routes that are 'good' options mainly due to a good evaluation in terms of low motorized traffic volumes are more likely to be found.

35 3.2.6. Utility values

The marginal travel time and travel distance utilities are chosen as $\beta_{\text {trav }, b}=$ $-6.0 \mathrm{utils} / \mathrm{h}$ and $\beta_{d, b}=-0.0004 \mathrm{utils} / \mathrm{m}$, such that an additional meter yields the same utility offset as an additional travel time of $1 \mathrm{~s}$ when traveling at a speed of $15 \mathrm{~km} / \mathrm{h}$.

The parameter $\beta_{\text {inf (a) }}$ is intended to pick up aspects of continuous and wellmarked cycling infrastructure (cf. section 2) of a given link $a$. Its value is computed as

$$
\beta_{i n f(a)}=\beta_{i n f(a)}^{\max } \cdot(1-\inf (a))
$$

440 where $\beta_{\inf (a)}^{\max }=-0.0002 \mathrm{utils} / \mathrm{m}$, such that at most half of the marginal utility of distance can be added. $\inf (a)$ is dependent on the highway type and the existence of a dedicated cycling infrastructure. The values of this term are stated for some highway types in table 2, It can be seen that for a dedicated cycleway no (negative) utility offset is added because these infrastructures are regarded as very accommodating for cyclists. A primary road without any form of cycling infrastructure, by contrast, receives the maximum additional utility offset.

The marginal utility of comfort $\beta_{\operatorname{comf}(a)}$ is intended to reflect pavement conditions and riding smoothness (cf. section 2): The smoother the surface, the higher the utility value. Just like for the marginal utility of infrastructure, the maximum value of the marginal utility of comfort is chosen as $\beta_{\operatorname{comf}(a)}^{\max }=$ -0.0002 utils $/ \mathrm{m}$. The effectively used marginal utility of comfort for a link $a$ is computed as

$$
\beta_{\operatorname{comf}(a)}=\beta_{\operatorname{comf}(a)}^{\max } \cdot(1-\operatorname{comf}(a))
$$


Table 2: Infrastructure factor for different highway types (not all values shown).

\begin{tabular}{ll}
\hline Highway type & Infrastructure factor \\
\hline primary & $0 \%$ \\
primary with dedicated cycling infrastructure & $95 \%$ \\
secondary & $30 \%$ \\
secondary with dedicated cycling infrastructure & $95 \%$ \\
cycleway & $100 \%$ \\
\hline
\end{tabular}

where the value $\operatorname{comf}(a)$ of a link $a$ with a specific surface type is the same as the value of the surface-based speed factors of table 1

The marginal utility of gradient $\beta_{\operatorname{grad}(a)}$ considers the fact that cyclists try to avoid uphill slopes (cf. section 22. Its value is selected as $\beta_{\operatorname{grad}(a)}=$ -0.004 utils $/(\mathrm{m} / 100 \mathrm{~m})$. This means that an agent climbing a hill of $10 \mathrm{~m}$ height receives the same utility offset as one who takes a detour of $500 \mathrm{~m}$. This is loosely based on the marginal rates of substitution (MRS) reported by Hood et al. [6. Broach et al. [11] find cyclists to be willing to pedal 2,750 m (or 2,190 m when commuting) on flat terrain if the alternative is $1,609 \mathrm{~m}$ (i.e. 1 mile) with an upslope of $2 \%$ to $4 \%$, which confirms the aforementioned substitution rate.

The value of the marginal utility of an interaction with a motorized vehicle (cf. section 3.2.4 is chosen as $\beta_{\text {motor }}=-0.004$ utils. This means that the utility offset incurred by traveling for one hour on links with a high motorized traffic load (e.g. 1200 veh/h, i.e. one car every 3 seconds) compared to traveling on roads with low motorized traffic loads (e.g. 200 veh/h, i.e. one car every 18 seconds) is the same as the utility offset of a detour of $20 \mathrm{~min}$. These values are informed by findings of Sener et al. [5].

\section{Results}

In this section, the adaptations described in section 3.2 are tested in an illustrative scenario. This scenario is based on the so-called equil scenario [8], 
which - in its original version - contains nine alternative, but fully identical routes. The corresponding network is depicted in figure 2, For testing the

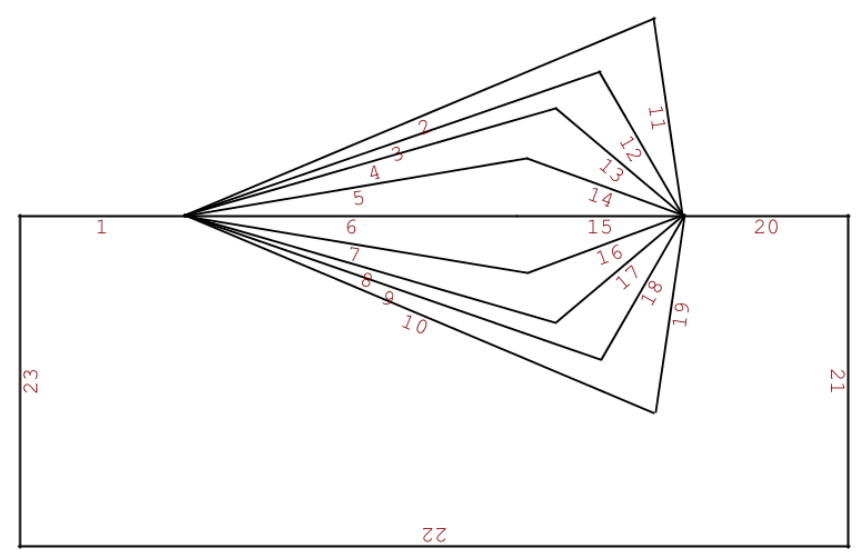

Figure 2: Equil network with link identifiers

470 of the nine alternative routes increase from the central route to the outer routes: The central route (links 6 and 15 in figure 2) has a length of 15,000 m between the nodes where the nine alternative routes fork and merge again, while the other routes - ascending toward the outermost routes - possess lengths of $15,500 \mathrm{~m}$, $16,000 \mathrm{~m}, 17,000 \mathrm{~m}$, and 18,000 m. All links are characterized as primary roads.

1,200 agents travel from the left of the scenario to the right, starting their trip with an offset of 3 seconds to their predecessor, with the first agent starting at 9:00. The only choice they can take is selecting one of the nine alternative routes, i.e. no innovative strategy module other than the routing module is activated for this test. Because of the random components of the router, agents may also choose a non-optimal route, while the probability to do so decreases with the extent to which that route deviates from the optimum with regard to utility as in equations 6 and 7 . Over the course of iterations, scoring and plan selection effect that the optimal route with respect to the coring function

\footnotetext{
8 This scenario can be run by invoking RunBicycleExample; cf. http://matsim.org/ javadoc $\rightarrow$ bicycle $\rightarrow$ RunBicycleExample.
} 


\subsection{Infrastructure-related attributes}

First, the impact of the consideration of various infrastructure-related attributes on the behavior of cyclists is analyzed. The interaction of cyclists with motorized traffic is not yet taken into account in this section. All experiments are simulated over 10 iterations.

Figure $3 \mathrm{a}$ represents the base case where all links have identical properties (except different link lengths as described above). Expectedly, agents tend to choose the more central routes because of their better travel utility values, which are due to lower travel times on these routes.

In figure $3 \mathrm{~b}$, the five central links are converted into streets that are paved with cobblestones. As described in section 3.2.2 this reduces travel speeds and comfort. As a consequence, it can be seen that agents avoid the routes with cobblestones quite clearly and divert to the outer (non-cobblestone) routes. Among these four outer routes, the two shorter ones (i.e. the routes via link 2 and via link 9, respectively (cf. figure 2, both with a length of 17,000 m) are more strongly used than the two longer, outermost route alternatives (each with a length of 18,000 m): As can be seen in table 3. after 10 iterations, 545 and 531 agents use the shorter routes via link 3 and link 9, respectively, while only 47 and 48 agents use the somewhat longer routes (via link 2 and link 10), respectively. Of the routes that contain links paved with cobblestones, only the route via link 6 , the absolute shortest in the network (with a length of $15,000 \mathrm{~m}$ ), is used by 29 agents. As can also be seen in table 3 the router immediately (i.e. already in iteration 0) finds quite exactly those route options that prevail over multiple iteration.

Next, the five central links are designated as pedestrian zones (cf. figure 3c), where cyclists can only travel with reduced speeds (cf. section 3.2.2). As explained in section 3.2 .3 , they also obtain a somewhat lower infrastructure utility when riding through a pedestrian zone. As in the case before, agents quite clearly divert to the outer (non-pedestrianized routes) and accept longer dis- 


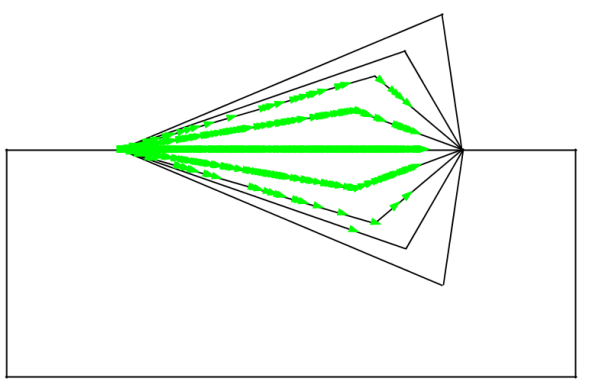

(a) Base Case: All links designated as highway $=$ Primary

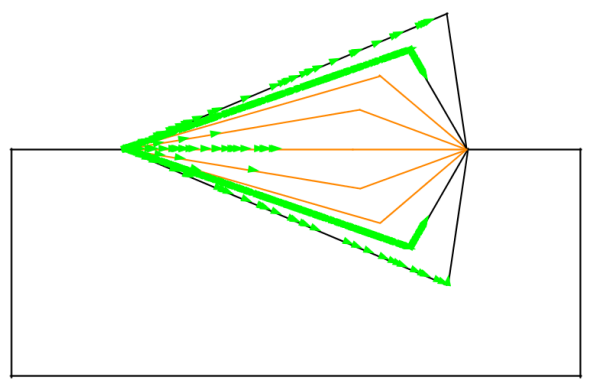

(c) Five central links are designated as highway $=$ pedestrian.

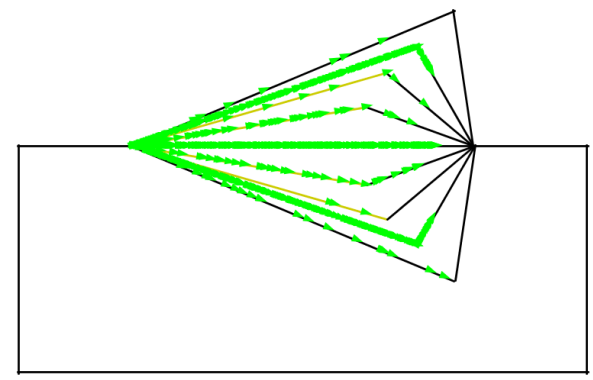

(e) Five central links have a gradient of $3 \%$.

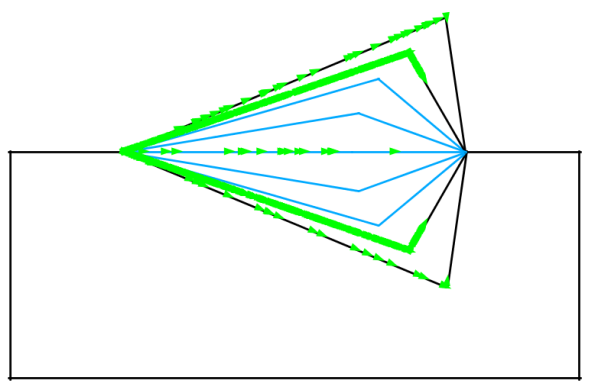

(b) Five central links are paved with cobblestones.

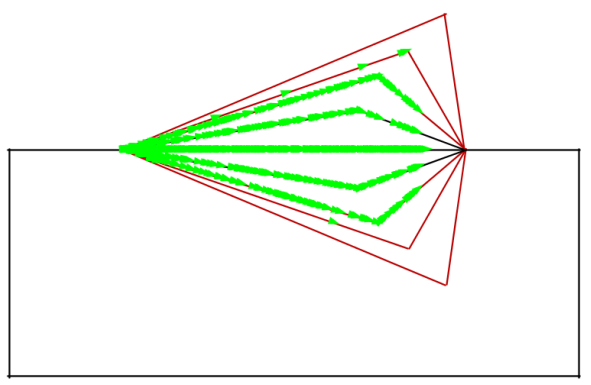

(d) Six outer links have a bicycle lane (i.e. designated as cycleway $=$ lane $)$.

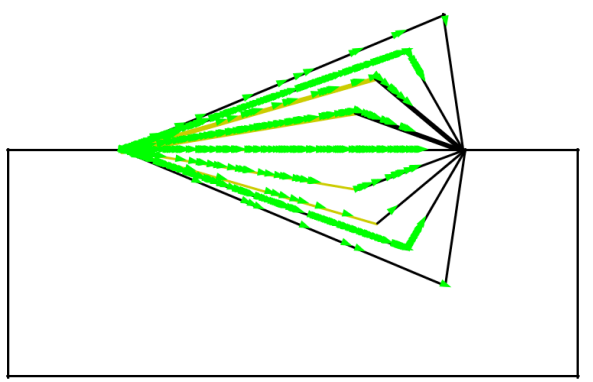

(f) Five central links have a gradient of $3 \%$, two upper of them with a bicycle lane.

Figure 3: Experiments with illustrative scenario for different infrastructure-related attributes. 
the pedestrian zones is associated with. The higher travel times in the pedestrian zones can be observed in figure $3 \mathrm{c}$. Agents who use the central routes have made it significantly less far on the network than the agents who travel on the outer routes.

520 ture. In table 4 it can be observed that, over the course of the iterations, the preference for the two shortest among the six routes with bicycle lanes becomes clearer.

Table 3: Bicycle counts when some links have cobblestones (marked with (C)).

\begin{tabular}{lrrr}
\hline Link & It. 0 & It. 10 & It. 100 \\
\hline 2 & 46 & 47 & 9 \\
3 & 527 & 545 & 594 \\
$4(\mathrm{C})$ & 0 & 0 & 0 \\
$5(\mathrm{C})$ & 0 & 0 & 0 \\
$6(\mathrm{C})$ & 21 & 29 & 26 \\
$7(\mathrm{C})$ & 0 & 0 & 0 \\
$8(\mathrm{C})$ & 0 & 0 & 0 \\
9 & 566 & 531 & 565 \\
10 & 40 & 48 & 6 \\
\hline
\end{tabular}

Table 4: Bicycle counts when some links have bicycle lanes (marked with (L)).

\begin{tabular}{lrrr}
\hline Link & It. 0 & It. 10 & It. 100 \\
\hline $2(\mathrm{~L})$ & 0 & 0 & 0 \\
$3(\mathrm{~L})$ & 7 & 7 & 14 \\
$4(\mathrm{~L})$ & 127 & 152 & 357 \\
5 & 199 & 156 & 25 \\
6 & 558 & 569 & 392 \\
7 & 171 & 158 & 38 \\
$8(\mathrm{~L})$ & 181 & 150 & 354 \\
$9(\mathrm{~L})$ & 7 & 7 & 10 \\
$10(\mathrm{~L})$ & 0 & 1 & 0 \\
\hline
\end{tabular}

In figure 3e, a gradient of $3 \%$ is introduced on the five central links by increasing the elevation value of the coordinate of their respective end nodes. As explained in section 3.2 .2 , the negative slopes (downhill) on the subsequent links do not have an impact on travel utilities in the current implementation. As expected, many agents avoid the slopes and divert to the outer routes and accept 
longer distances and travel times rather than having to travel with reduced speeds and incurring a negative offset in their gradient-based utility.

Figure [3] shows a tradeoff situation between link length, gradient, and infrastructure. The addition of bicycle lanes on the two upper links with gradients helps counteracting the unattractiveness of these uphill links (cf. figure 3e), on which agents also have to cover a somewhat longer distance than on the central route. As such, it can be observed that some agents use these routes after the addition of bicycle lanes.

\subsection{Traffic-related attributes}

Now, the reaction of cyclists to interaction with motorized traffic as they have experienced it in the simulation of the synthetic reality (cf. section 3) is taken into account. Such an interaction with a motorized vehicle can, for instance, consist in a cyclist being overtaken by a car, potentially on a street with no dedicated cycling infrastructure.

By applying the amendments described in section 3.2.4. cyclists translate such events into their scoring, which - over the course of multiple iteration in the co-evolutionary simulation framework - will make the selection of routes with a lot of motorized traffic less likely.

While for some choice situations related to infrastructure properties (especially those choice situations where the best option was clearly distinguishable; cf. section 4.1), the router managed to find the preferred route options immediately (i.e. in iteration 0), this cannot be the case in the current experiment, which addresses events that are emergent from the simulation of the physical reality. As such, a corresponding adaptation of agents' behavior can only be expected after a number of iterations, in which agents have 'experienced' these events (the passings) in the simulation of the synthetic reality.

In figure 4 two simulation runs with each 100 iterations are compared. The first run (cf. figure 4a) uses the settings that were used to create the results in figure $3 \mathrm{a}$ (using 100 iterations instead of 10 iteration being the sole alteration). In the second run (cf. figure 4b, a synthetic MotorizedInteractionEvent is 


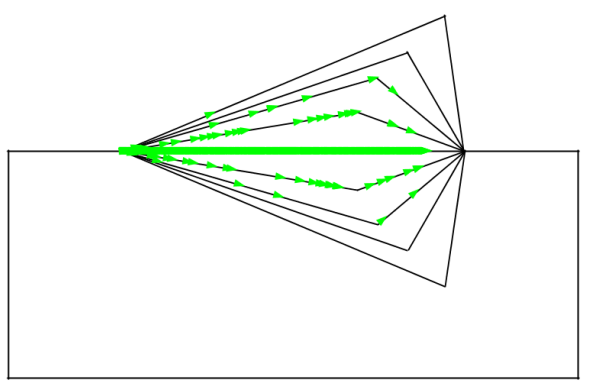

(a) Base network.

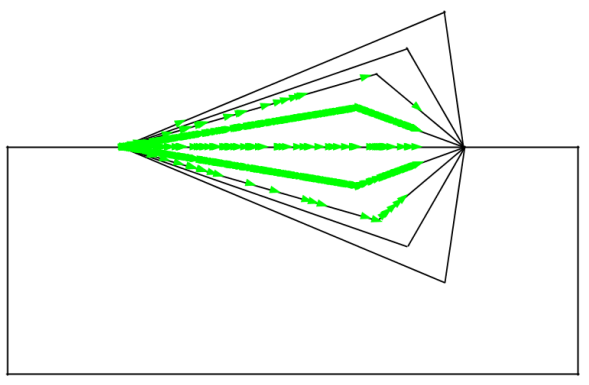

(b) Base network with 1200 cars/h on central route.

Figure 4: Experiments with illustrative scenario for interaction with motorized traffic.

thrown every 3 seconds on link 6 (i.e. the first link of the central route, cf. figure 2), which can be interpreted as a motorized traffic load of 1,200 cars/h on this link. For each such event that happens while a cycling agent traverses this link, this agent incurs a score offset of $\beta_{\text {motor }}=-0.004$ utils as described in section 3.2.4. As can be seen in the figure $4 \mathrm{~b}$, after 100 iterations, agents avoid the central route, while this route is the preferred option if there is no motorized traffic on this route (cf. figure 4a). Table 6 shows agents' adaptation process to the traffic situation in the two experiments over the course of multiple iterations.

Table 5: Bicycle counts on base network (no other vehicles present).

\begin{tabular}{lrrr}
\hline Link & It. 0 & It. 10 & It. 100 \\
\hline 2 & 0 & 0 & 0 \\
3 & 1 & 0 & 0 \\
4 & 34 & 44 & 9 \\
5 & 184 & 187 & 38 \\
6 & 744 & 729 & 1101 \\
7 & 200 & 194 & 42 \\
8 & 37 & 43 & 9 \\
9 & 3 & 0 & 0 \\
10 & 0 & 0 & 0 \\
\hline
\end{tabular}

Table 6: Bicycle counts on base network (1200 cars/h on route marked with (C)).

\begin{tabular}{lrrr}
\hline Link & It. 0 & It. 10 & It. 100 \\
\hline 2 & 0 & 0 & 0 \\
3 & 1 & 0 & 0 \\
4 & 34 & 44 & 30 \\
5 & 184 & 260 & 476 \\
$6(\mathrm{C})$ & 744 & 542 & 134 \\
7 & 200 & 270 & 515 \\
8 & 37 & 53 & 44 \\
9 & 0 & 3 & 0 \\
10 & 0 & 0 & 0 \\
\hline
\end{tabular}




\section{Discussion and outlook}

In this study, different amendments to the agent-based transport simulation framework MATSim were introduced in order to model and analyze bicycle traffic more realistically.

The network generation procedure has been enriched by a method that retrieves elevation data from an open data source. Furthermore, additional cycling-relevant infrastructure attributes like road category and existence of dedicated bicycle infrastructure are included into the network, taking advantage of a recent amendment to MATSim objects, which allows them to contain arbitrary additional attributes.

For the computation of travel times for cyclists, features of the network (incl. aforementioned additional attributes) as well as gradients and road surface type are observed.

Similarly, the scoring function has been adjusted to take into account road gradients, cycling infrastructure, and riding comfort apart from travel time and distance that are already part of the default version of the MATSim utility function. Furthermore, an explicit evaluation of the interaction of cyclists with motorized vehicles is included into the scoring function, picking up on the effect that cyclists tend to avoid streets were they are affected by many passings by motorized vehicles. By this, full advantage is taken of the fact that bicycles are simulated explicitly on the network.

Similarly, MATSim's routing module was adjusted to enable it to find reasonable route options for cyclists, which can then be simulated in the synthetic reality and evaluated based on the aforementioned scoring function. Inspired by an evolutionary computing perspective, a randomizing router was implemented including the attributes that are part of the scoring function except the interaction with other traffic, which is, by definition, simulation-emergent and thus unknown to the router. It is discussed why this approach is both feasible in terms of generating suitable routing results and also consistent with the concept of MATSim's co-evolutionary simulation approach. 
The specific values of the marginal utilities of infrastructure attributes and choice-relevant events have been informed by empirical literature values and described.

Using an illustrative scenario, it was shown that the amendments for bicycle traffic introduced in this study lead to reasonable and expected results. Realworld transport simulation scenarios should become more realistic when the new methods are applied to them.

Similar to those attributes that are already included, also additional attributes mentioned in section 2, e.g. bus stops, parking facilities, and junctions can be included if desired. It seems feasible that these attributes can also be sourced from OSM data.

Furthermore, and probably more interesting from the perspective of an agent-based simulation framework, person- and/or vehicle-specific characteristics can be considered in the scoring function. For instance, researchers found that women tend to avoid slopes more strongly than men and commuters stick more strongly to the shortest route (cf. section 2). Both phenomena can be modeled in MATSim, the former by assigning demographics to agents and adjusting the scoring function based on this, the latter by observing the sequence ${ }_{620}$ of activities in the daily plans of agents and making decisions dependent on subsequent activities (e.g. work vs. leisure activities). The technical implementation of this can be oriented on the adjustment of the scoring process for the interaction of cyclists with motorized traffic shown in this paper.

Similarly, it is possible to further distinguish between different types of bi625 cycles (e.g. race bicycles vs. beach cruisers) with their typical maximum speeds.

Since only openly accessible input data have been used, the approach is reproducible and easy to transfer to other spatial contexts. Future studies to use the described approaches for policy evaluation studies are underway. 


\section{Acknowledgement}

630 MATSim objects, which can now possess user-specified attributes. This allows to add information on cycleway types and surface types directly to the network link objects without having to carry along additional specification files. Support by Stiftung Mercator, which partly funded this study in the context of the thank two anonymous reviewers for their helpful comments.

\section{References}

[1] G. Menghini, N. Carrasco, N. Schüssler, K. Axhausen, Route choice of cyclists in Zurich, Transportation Research Part A 44 (2009) 754-765.

[2] Z. Li, W. Wang, P. Liu, D. Ragland, Physical environments influencing bicyclists perception of comfort on separated and on-street bicycle facilities, Transportation Research Part D 17 (2012) 256-261.

[3] D. Milakis, K. Athanasopoulos, What about people in cycle network planning? Applying participative multicriteria GIS analysis in the case of the Athens metropolitan cycle network, Journal of Transport Geography 35 (2014) 120-129.

[4] M. Ehrgott, J. Y. Wang, A. Raith, C. van Houtte, A bi-objective cyclist route choice model, Transportation Research Part A 46 (2011) 652-663.

[5] I. Sener, N. Eluru, C. Bhat, An analysis of bicycle route choice preferences in Texas, US, Transportation 36 (2009) 511-539.

[6] J. Hood, E. Sall, B. Charlton, A GPS-based bicycle route choice model for San Francisco, California, Transportation Letters (3) (2011) 63-75. doi: 10.3328/TL. 2011.03.01.63-75. 
[7] G.-A. Ahrens, F. Ließke, R. Wittwer, S. Hubrich, S. Wittig, Tabellenbericht zum Forschungsprojekt 'Mobilität in Städten - SrV 2013' in Berlin, http://www.stadtentwicklung.berlin.de/verkehr/ politik_planung/zahlen_fakten/mobilitaet_2013/ (2014).

[8] A. Horni, K. Nagel, K. W. Axhausen (Eds.), The Multi-Agent Transport Simulation MATSim, Ubiquity, London, 2016. doi:10.5334/baw.

[9] C. Dobler, G. Lämmel, The multi-modal contribution, in: Horni et al. [8], Ch. 21. doi:10.5334/baw.

[10] D. Ziemke, S. Metzler, K. Nagel, Modeling bicycle traffic in an agentbased transport simulation, Procedia Computer Science 109 (2017) 923928. doi:10.1016/j.procs.2017.05.424.

[11] J. Broach, J. Dill, J. Gliebe, Where do cyclists ride? a route choice model developed with revealed preference GPS data, Transportation Research Part A: Policy and Practice 46 (10) (2012) 1730-1740. doi:10.1016/j. tra.2012.07.005.

[12] L. Bai, P. Liu, C.-Y. Chan, Z. Li, Estimating level of service of mid-block bicycle lanes considering mixed traffic flow, Transportation Research Part A: Policy and Practice 101 (2017) 203-217. doi:10.1016/j.tra.2017. 04.031

[13] F. Ayachi, J. Dorey, C. Guastavino, Identifying factors of bicycle comfort: An online survey with enthusiast cyclists, Applied Ergonomics 46 (2015) $124-136$.

[14] B. Landis, V. Vattikuti, M. Brannick, Real-time human perceptions: Toward a bicycle level of service, Transportation Research Record (1578) (1997) 119-126.

[15] C. Hölzel, F. Höchtl, V. Senner, Cycling comfort on different road surfaces, Procedia Engineering 34 (2012) 479-484. 
[16] M. Bíl, R. Andrášik, J. Kubuček, How comfortable are your cycling tracks? A new method for objective bicycle vibration measurement, Transportation Research Part C 56 (2015) 415-425.

[17] C. Hebenstreit, M. Fellendorf, A dynamic bike sharing module for agentbased transport simulation, within multimodal context, Procedia Computer Science 130 (2018) 65-72.

[18] S. Flügel, N. Hulleberg, A. Fyhri, C. Weber, G. Ævarsson, Empirical speed models for cycling in the Oslo road network, Transportationdoi:10.1007/ s11116-017-9841-8.

[19] K. Nagel, B. Kickhöfer, J. W. Joubert, Heterogeneous tolls and values of time in multi-agent transport simulation, in: Procedia Computer Science [22], pp. 762-768 (2014) 762-768. doi:10.1016/j.procs.2014.05.488

[20] K. Nagel, B. Kickhöfer, A. Horni, D. Charypar, A closer look at scoring, in: Horni et al. [8], Ch. 3. doi:10.5334/baw

[21] E. Dijkstra, A note on two problems in connexion with graphs, Numerische Mathematik 1 (1959) 269-271.

[22] K. Nagel, B. Kickhöfer, J. W. Joubert, Heterogeneous tolls and values of time in multi-agent transport simulation, Procedia Computer Science 32 (2014) 762-768. doi:10.1016/j.procs.2014.05.488.

[23] K. Nagel, G. Flötteröd, Agent-based traffic assignment: Going from trips to behavioural travelers, in: R. Pendyala, C. Bhat (Eds.), Travel Behaviour Research in an Evolving World - Selected papers from the 12th international conference on travel behaviour research, International Association for Travel Behaviour Research, 2012, pp. 261-294.

[24] OpenStreetMap, http://www.openstreetmap.org (last accessed on 25 June 2018). 
[25] European Digital Elevation Model, http://data.eox.at/eudem (last accessed on 25 June 2018).

[26] J. W. Joubert, Multi-agent model of route choice when vehicles are sensitive to road grade, Procedia Computer Science 109C (2017) 869-874, the 6th International Workshop on Agent-based Mobility, Traffic and Transportation Models, Methodologies and Applications (ABMTRANS). doi:10.1016/j.procs.2017.05.402.

[27] A. Agarwal, M. Zilske, K. Rao, K. Nagel, An elegant and computationally efficient approach for heterogeneous traffic modelling using agent based घ simulation, Procedia Computer Science 52 (C) (2015) 962-967. doi:10. $1016 / j$.procs. 2015.05 .173 